\title{
Scanning tumeling microscopy on rough surfaces: Deconvolution of constant current images
}

\author{
G. Reiss, F. Schneider, J. Vancea, and H. Hofmann \\ Institut fur Ang. Physik III, Universität Regensburg Universitätsstrasse 31, D-8400 Regensburg, \\ Federal Repubic of Germany
}

(Received 2 April 1990; accepted for publication 19 June 1990)

\begin{abstract}
This letter critically discusses the topographical information obtained by scanning tunneling microscopy (STM) on surfaces with a mesoscopic roughness, i.e, in the range of some nm's. In a foregoing publication [J. Appl. Phys. 67, 1156 (1990)], we already treated the evaluation of constant current images based on the knowledge of the real surface and the shape of the tunneling tip ("tip shape limited resolution"). Now we deal with the invers problem: the reconstruction of the real surface topography based on the corresponding STM image and the tip shape, using a simple, straightforward formalism.
\end{abstract}

The resolution of scanning tunneling microscopy (STM) on mesoscopically rough surfaces is clearly limited by the corresponding shape of the tunneling tip. ${ }^{1-6}$ For fiat surfaces only the front atoms of the tip are important. ${ }^{7,8}$ In our case, however, the mesoscopic (i.e., nm range) shape of the tunneling tip must be regarded. For potentiometric STM measurements, this has been qualitatively lined out recently by Pelz and Koch."

In Ref. I we have shown that only surface features of width and depth smaller than (roughly spoken) the tip's front shape can be resolved. A known surface topography can be simply convoluted to an STM image if the tip shape is known. The limits of resoiution can be expressed quantitatively by an inequality. ${ }^{1}$

The invers problem, however, seems to be of more practical interest: deconvolution of the real surface topography using the "as-measured" STM result and a tip shape evaluated by electron microscopy or directy from the STM image. Consider an STM topography represented by the height function $i(x, y)(x, y$ : in plane coordinates) produced with a tip described by $t(x, y)$, which scans a real surface $s(x, y)$. The task now is to find $s(x, y)$ using $i(x, y)$ and $t(x, y)$. The problem is sketched in Fig. 1.

If tunneling between the tip $t$ and the surfaces occurs at a certain point $S=\left[x^{\prime}, y^{\prime}, s\left(x^{\prime}, y^{\prime}\right)\right]$, the corresponding image point $I=\left[x_{0}, y_{0}, i\left(x_{0}, y_{0}\right)\right]$ in general has neither the same in-plane coordinates $\left(x^{\prime} \neq x_{0}, y^{\prime} \neq y_{0}\right)$ nor the same height $\left[s\left(x^{\prime}, y^{\prime}\right) \neq i\left(x_{0}, y_{0}\right)\right]$. Based on the knowledge of $i(x, y)$ and $t(x, y)$, we thus have to find all points of tunneling contact, i.e., the transformation

$$
\begin{aligned}
& x_{0} \rightarrow x^{\prime}, \\
& y_{0} \rightarrow y^{\prime}, \\
& i\left(x_{0}, y_{0}\right) \rightarrow s\left(x^{\prime}, y^{\prime}\right) .
\end{aligned}
$$

This transformed, new set of points then represents the real surface topography. Since the tunneling distance is nuch smaller than the features of tip and surface, ${ }^{7,8}$ the transformation can be performed in the following manner: Clearly, the tip and the surface show parallel tangential planes at the point of tunneling contact (Fig. 1). If the tip scans the surface, the corresponding change in the image is given by the movement of the mimimum of the tip. The differential change in the tip position, however, can be described using the slopes of the tangential plane at the point of turneling contact ( $S$ in Fig. 1). Consequently, the local tangents of the image in $I$ will be parallel to those of the tip and the surface in $S$ (see Fig. 1). For convenience, we always chose the tip $t(x, y)$ symmetric to the vertical axis with minimum in $(0,0,0)$. The point of tunneling contact on the tip is denoted by $T=[\Delta x, \Delta y, t(\Delta x, \Delta y)]$. From these statements and with the common definition of normal vectors we therefore obtain

$$
\mathbf{n}_{i}\left(x_{0}, y_{0}\right)=\left(\begin{array}{c}
\left.\frac{\partial i}{\partial x}\right|_{x=x_{0}} \\
\left.\frac{\partial i}{\partial y}\right|_{y=y_{0}}
\end{array}|=| \begin{array}{c}
\left.\frac{\partial t}{\partial x}\right|_{x=\Delta x} \\
\left.\frac{\partial t}{\partial y}\right|_{y=\Delta y} \\
1
\end{array}\right)=\mathbf{n}_{i}(\Delta x, \Delta y)
$$

Using Eq. (2), the point of tunneling contact on the tip, $T$, can be evaluated from the local slope of the image. Since, in turn, the image point $I$ corresponds to the minimum of the tip (see Fig. 1), the displacement between the real surface

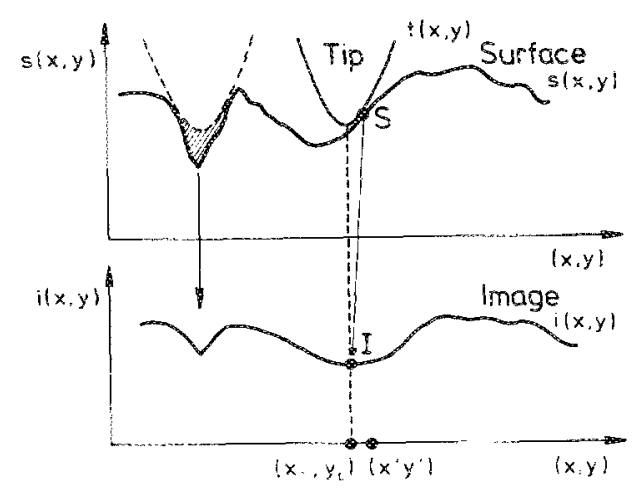

FIG. 1. Sketch of the typical situation of STM measurements on mesoscopically rough surfaces; the surface $s(x, y)$ is convoluted with the tip $t(x, y)$ to the image $i(x, y)$. The shaded region of the surface cannot be reached by the tip. 
point $S$ and this image point $L$ is given by the corresponding coordinates of the tip. Thus the job will be (a) caiculate $n_{i}$ for each point $I=\left[x_{0}, y_{0}, i\left(x_{0}, y_{0}\right)\right]$ of the image $i(x, y)$; (b) find the corresponding points $T=[\Delta x, \Delta y, t(\Delta x, \Delta y)]$ on the tip, where Eq. (2) is satisfied; (c) the corresponding points of tunneling contact on the surface then will be given by

$$
S=\left[x_{0}+\Delta x_{2} y_{0}+\Delta y, i\left(x_{0,}, y_{0}\right)+t(\Delta x, \Delta y)\right] \text {. }
$$

The transformation of Eq. (1), therefore, is given by

$$
\begin{aligned}
& x_{0} \rightarrow x^{\prime}:=x_{0}+\Delta x \\
& y_{0} \rightarrow y^{\prime}:=y_{0}+\Delta y \\
& i\left(x_{0}, y_{0}\right) \rightarrow s\left(x^{\prime}, y^{\prime}\right):=i\left(x_{0}, y_{0}\right)+i(\Delta x, \Delta y) .
\end{aligned}
$$

The set of points $S$ calculated with this procedure then represents those areas of the real surface, which had been in tunneling contact during imaging, i.e., which effectively can be "seen" by the tip. The remaining parts of the surface (the bottom of the deep hole sketched in Fig. 1, for example), i.e., those "depopulated" by this formalism, have not been really imaged and therefore cannot be specified. We only can state that these "black holes" show smaller features than the tip shape. Any further assumpions are not realistic. This procedure thus provides a rather simple method for the reconstruction of realistic surface topographies. The numeric software claims some refinement in order to exclude artifacts produced, for example, by noise or by the bottom of typical triangular hollows (see Refs. $1,2)$. The validity of this approach clearly is restricted to rough surfaces, i.e., the magnitude of the corrugations should remarkably exceed typical atomic dimensions.

The frst application concerns the surface of a Au-Nb-Au triple-layered thin fim on $\mathrm{Si}(100)$ already discussed in Refs. 1, 2; transmission electron microscopy (TEM) cross-section images revealed an island-like struciure of the upper Au film. This, however, could not be resolved by STM imaging due to the tip shape limited resolution, 'the features of the real surface and those of the tip are of the same "mesoscopic" order of magnitude (i.e., of some nm's). In Ref. 1 , the "worst case" limits for the tip shape have been estimated from STM images of Ali-covered $\mathrm{NaCl}(100)$ cleavage steps (radius of curvature: $5 \mathrm{~nm}$, tip cone angle: $30^{\circ}$ ).

A more useful estimation of the particular tip used for individual images can be performed via these images themselves. The tip cone angle is always smaller than the maximum observed slope shown by the STM images. Additionally the tip radius must be smaller than the minimum racius of curvature of convex structures observed by STM. The tip function $t(x, y)$ can therefore be evaluated by a careful fitting to various cross sections of the original STM image $i(x, y)$. The "as-imaged" STM topography of the Au-Nb-Au film is shown in Fig. 2 (a); Fig. 2(b) shows the results of tip evaluation (inset) and (real) surface deconvolution. As already discussed in Ref. 1, the STM image [Fig. 2(a)] suggests a rough but continuous surface in contradiction with TEM results. The evaluation of the tip shape using this picture [Fig. 2(a)] revealed the form in-
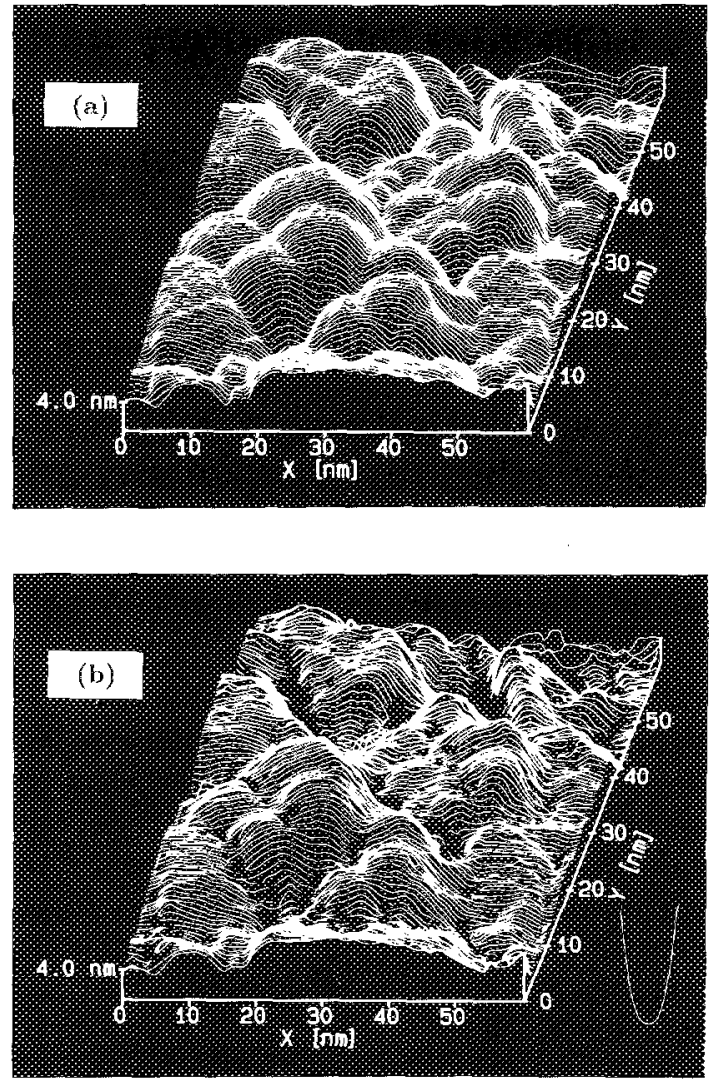

MG. 2. (a) Original STM image of the surface of a Au-Nb-Au triplelayered thin film on $\mathrm{Si}(100)$ with rough but continuous topography. (b) Deconvoluted real surface topography showing stronger features than the original image (a) and "Black holes," (inset) shape of the tunneling tip (same scals).

dicated in Fig. 2(b): the radius of curvature is about $2-3$ $\mathrm{nm}$, i.e., smaller than obtained by imaging $\mathrm{NaCl}(100)$ cleavage steps. The cone angle amounts to about $30^{\circ}$ in agreement with our previous value. ${ }^{1}$ th should be noted that from the same surfaces smooth images could be obtained if very blunt tips were used. A rough estimation leads to possible tip radii of up to some hundreds of $\AA$ 's.

In contrast with the STM image [Fig. 2(a)], the deconvoluted surface of the Au-Nb-Au film [Fig. 2(b)] corresponds rather to a thin film just before coalescence in agreement with the TEM results: The smooth rolling hiil structure of the unconvoluted image [Fig. 2(a)] is strongly removed; the recalculation [Fig. 2(b)] shows large "black holes" (about 10\% of the whole area) surrounding steeper hills than in the original image [Fig. 2(a)]. As already mentioned, the unidentified regions correspond to those parts of the real surface the tip has not been in tumeling contact with. Thus these areas cannot be recalculated. In any case, however, they consist of steep and narrow structures, at least smaller than the shape of the tunneling tip. An interpretation of the surface topography using only the STM image of Fig. 2(a) thus rather leads to wrong results. This first example, therefore, clearly illustrates the reliability of the results supplied by the formalism discussed before. On the other hand, this specific exampie shows extremely critical topographies, i.e, features of the same lateral and vertical extension as the tunneling tip. 

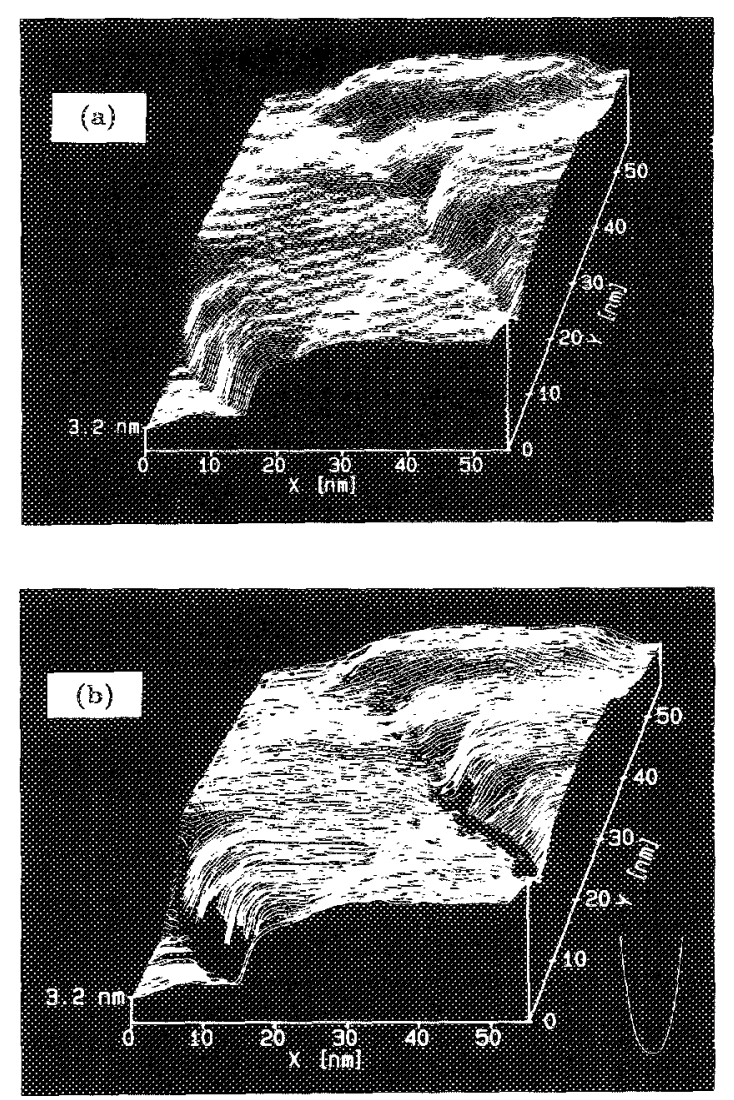

FIG. 3. (a) Original STM inage of the topography of a Au sphere (1.5 $\mathrm{mm}$ in diameter) with flat plateaus separated by steep steps. (b) Deconvoluted surface topography showing mainly the same features as the STM image except some regions in the vicinity of the large steps of $(a)$, (inset) shape of the tunneling tip (same scale).

Our second example therefore deals with a surface not as strongly structured as the foregoing topography; small Au spheres of $1.5 \mathrm{~mm}$ in diameter have been produced by melting. These spheres are strongly facetted, i.e., exhibit large, optically flat regions. ${ }^{10}$ A typical STM image of these areas is given in Fig. 3(a): in agreement with the results of Schneir et al., ${ }^{10}$ the facets show large fiat plateaus, where only monoatonic steps can be resolved. These plateaus, however, are separated by larger steps of various height."

Similar to the foregoing example, the tip shape can be evaluated using the strongest features of the image [i.e., the large step in the lower left corner of Fig. 3(a)]. The result is shown in Fig. 3(b) together with the deconvoluted topography. The tip function obtained from the image [Fig. $3(a)]$ is aimost identical with the first example: radius of curvature $2-3 \mathrm{~nm}$, cone angle about $30^{\circ}$. This evaluation of the tip shape from each individual image therefore seems to have a high degree of reliability. Using this tip shape and the STM image of Fig. 3(a), the surface shown in Fig. $3(b)$ was calculated. In contrast with the strongly featured surface of Fig. 2, this example shows less dramatic effects. The main, flat regions of the image remain unchanged. Only about $5 \%$ of the surface located at the bottom of the two steep steps cannot be resolved.

In the case of large, flat features, the STM images therefore provide reliable information about the real surface topography.

In summary, we have presented an attempt to obtain realistic topographies of mescscopically rough surfaces from as-measured STM images. Based on the STM image, the tip furction can be properly evaluated. Using these image and tip functions, a straightforward formalism for the deconvolution of the real surface topography can be applied. This procedure naturally provides only those parts of the real surface the tip had been in tunneling contact with. The presented examples demonstrate the reliability of our method of deconvolution. Whereas the strongly featured image of an islandized thin film suggests a continuous surface, the deconvolution reveals island structures in agreement with TEM results. On the other hand, surfaces consisting of rather flat plateaus separated by steep steps are well reproduced by the STM image except some areas at the fianks of these steps. A noticeable deviation of the STM image from the real surface topography therefore must be suspected as soon as the dimensions (i.e., height and width) of the surface's features become comparable with the shape of the tunneling tip. For this case, the present method provides a straightorward procedure for the evaluation of realistic surface topographies.

We are indebted to $H$. Brückl and $R$. Lecheler for supplying STM measurements and to Dr. J. Zweck for TEM cross-section image.

${ }^{1}$ G. Reiss, J. Vancea, H. Wittmann, J. Zweck, and H. Hofmana, J. Appl. Phys. 67, 1156 (1990).

2J. Vancea, G. Reiss, F. Schneider, K. Baver, and H. Hofmann, Surf. Sci. $218,108(1989)$.

${ }^{3}$ J. K. Gimzewski, A. Humbert, J. G. Bednorz, and B. Reihl, Phys. Rev. Lett. 55, 951 (1985).

${ }^{4}$ A. Bartolomé, R. Garcia, L. Yázquez., and A. M. Baró, J. Microse. 152, 205 (1988)

${ }^{5}$ Ph. Niedermann and 0 . Fischer, $\searrow$. Microsc. 152, 93 (1988).

${ }^{6} \mathrm{M}$. Stedman, Int. Soc. Opt. Eng. 1009, 56 (1989).

7J. Tersoff and D. R. Hamann, Phys. Rev. Lett. 50, 1998 (1983).

${ }^{8}$ N. D. Lang, Phys. Rev. B 37, 10395 (1988).

${ }^{9}$ J. P. Pelz and R. H. Koch, Phys. Rev. B 41, 1212 (1990).

${ }^{10}$ J. Schneir, R. Sonnenfeld, O. Marti, P. K. Hansma, J. E. Demuth, and

K. J. Hamers, J. Appl. Phys. 63, 717 (1988).

${ }^{11} \mathrm{H}$. Brückl and R. Lecheler (umpublished). 